TRANSACTIONS OF THE

AMERICAN MATHEMATICAL SOCIETY

Volume 360, Number 7, July 2008, Pages 3921-3941

S 0002-9947(08)04448-6

Article electronically published on January 11, 2008

\title{
THE NONCOMMUTATIVE WIENER LEMMA, LINEAR INDEPENDENCE, AND SPECTRAL PROPERTIES OF THE ALGEBRA OF TIME-FREQUENCY SHIFT OPERATORS
}

\author{
RADU BALAN
}

\begin{abstract}
In this paper we analyze the Banach *-algebra of time-frequency shifts with absolutely summable coefficients. We prove a noncommutative version of the Wiener lemma. We also construct a faithful tracial state on this algebra which proves the algebra contains no compact operators. As a corollary we obtain a special case of the Heil-Ramanathan-Topiwala conjecture regarding linear independence of finitely many time-frequency shifts of one $L^{2}$ function. We also estimate the coefficient decay of the inverse of finite linear combinations of time-frequency shifts.
\end{abstract}

\section{INTRODUCTION}

The time-frequency representation of the Heisenberg group has received a lot of attention for the past 20 years with the advent of Gabor analysis. Many methods and techniques have been developed and a rich body of results has been obtained. For a nice account of such results we refer the reader to the excellent book [9].

For $t \in \mathbf{R}^{d}$ and $\omega \in \mathbf{R}^{d}$ we denote by $S_{t}$ the time shift operator, by $M_{\omega}$ the frequency shift operator, and by $U_{t, \omega}$ the time-frequency shift operator defined, respectively, by:

$$
\begin{aligned}
S_{t}: L^{p}\left(\mathbf{R}^{d}\right) \rightarrow L^{p}\left(\mathbf{R}^{d}\right) \quad, \quad S_{t} f(x)=f(x-t), \\
M_{\omega}: L^{p}\left(\mathbf{R}^{d}\right) \rightarrow L^{p}\left(\mathbf{R}^{d}\right) \quad, \quad M_{\omega} f(x)=e^{i \omega x} f(x), \\
U_{t, \omega}: L^{p}\left(\mathbf{R}^{d}\right) \rightarrow L^{p}\left(\mathbf{R}^{d}\right) \quad, \quad U_{t, \omega} f(x)=M_{\omega} S_{t} f(x)=e^{i \omega x} f(x-t) .
\end{aligned}
$$

For a Banach space $X$, we let $B(X)$ denote the Banach space of bounded operators on $X$ with the usual operator norm. Now consider the Banach algebra (see [22, 23] for definition and properties of Banach algebras) of time-frequency shifts on $L^{2}\left(\mathbf{R}^{d}\right)$ with absolutely summable coefficients:

$$
\mathcal{A}=\left\{T \in B\left(L^{2}\left(\mathbf{R}^{d}\right)\right)\left|T=\sum_{\lambda \in \mathbf{R}^{2 d}} c_{\lambda} U_{\lambda},\|T\|_{\mathcal{A}}:=\sum_{\lambda \in \mathbf{R}^{2 d}}\right| c_{\lambda} \mid<\infty\right\}
$$

Note the following: (i) for any $T \in \mathcal{A}$, the support $\operatorname{supp}(T)$ of its generating sequence $c, \operatorname{supp}(T)=\left\{\lambda \in \mathbf{R}^{2 d}, c_{\lambda} \neq 0\right\}$ is always a countable set; (ii) this support is not assumed to have any lattice structure.

Wiener's lemma states that if a periodic function $f$ has an absolutely convergent Fourier series and never vanishes, then its reciprocal $1 / f$ also has an absolutely

Received by the editors November 9, 2005 and, in revised form, October 3, 2006.

2000 Mathematics Subject Classification. Primary 43A20; Secondary 42C15, $46 \mathrm{H} 30$.

Key words and phrases. Time-frequency shifts, operator algebras, Wiener lemma, trace.

(C)2008 American Mathematical Society 3921 
convergent Fourier series. In Banach algebra language the same result can be restated as follows. Consider the Banach algebra

$$
\mathcal{A}_{W}=\left\{T \in B\left(L^{2}\left(\mathbf{R}^{d}\right)\right)\left|T=\sum_{n \in \mathbf{Z}^{d}} c_{n} S_{n},\|T\|_{\mathcal{A}_{W}}:=\sum_{n}\right| c_{n} \mid\right\}
$$

which is a $*$-subalgebra of $B\left(L^{2}\left(\mathbf{R}^{d}\right)\right)$. Then Wiener's lemma asserts that, if $T \in$ $\mathcal{A}_{W}$ is invertible in $B\left(L^{2}\left(\mathbf{R}^{d}\right)\right)$, then $T^{-1} \in \mathcal{A}_{W}$. Such algebras are called inverse closed (see e.g. [12).

Many generalizations of this result appeared in literature. We will mention here four extensions that set the context of our results.

The theory of almost periodic functions contains the following result. Consider the Banach algebra

$$
\mathcal{A}_{A P}=\left\{T \in B\left(L^{2}\left(\mathbf{R}^{d}\right)\right)\left|T=\sum_{t \in \mathbf{R}^{d}} c_{t} S_{t}\right|\|T\|_{\mathcal{A}_{A P}}:=\sum_{t \in \mathbf{R}^{d}}\left|c_{t}\right|<\infty\right\}
$$

of absolutely summable linear combinations of arbitrary real shifts. Then using the Bohr compactification of $\mathbf{R}^{d}([20])$, it follows that $\mathcal{A}_{A P}$ is an inverse closed algebra in $B\left(L^{2}\left(\mathbf{R}^{d}\right)\right)$. More explicitly, if $T=\sum_{\lambda \in \Lambda} c_{\lambda} S_{\lambda}$ for some countable subset $\Lambda \subset \mathbf{R}^{d}$, and $\sum_{\lambda \in \Lambda}\left|c_{\lambda}\right|<\infty$, then $T^{-1}=\sum_{\sigma \in \Sigma} d_{\sigma} S_{\sigma}$ for some (in general) other countable subset $\Sigma \subset \mathbf{R}^{d}$ and absolutely summable complex coefficients $\sum_{\sigma \in \Sigma}\left|d_{\sigma}\right|<\infty$. See also [26] for an extension to matrix valued almost periodic functions.

In the context of time-frequency analysis Gröchenig and Leinert [12] obtained a discrete noncommutative Wiener lemma as follows. Fix $\alpha, \beta>0$ and a subexponential weight $v$ (see section 2). We let $\mathcal{A}_{v ; G L}(\alpha, \beta)$ denote the operator algebra

$$
\begin{array}{r}
\mathcal{A}_{v ; G L}(\alpha, \beta)=\left\{T \in B\left(L^{2}\left(\mathbf{R}^{d}\right)\right)\left|T=\sum_{k, l \in \mathbf{Z}^{d}} a_{k, l} S_{\alpha k} M_{\beta l}\right|\|T\|_{\mathcal{A}_{v}}\right. \\
\left.:=\|\mathbf{a}\|_{1, v}:=\sum_{k, l \in \mathbf{Z}^{d}} v(k, l)\left|a_{k, l}\right|\right\}
\end{array}
$$

where $\|\mathbf{a}\|_{1, v}$ is the $v$-weighted $l^{1}$ norm of $\mathbf{a}$. Then, using Ludwig's theorem on symmetric group algebras of nilpotent groups (21]), the authors proved in [12] (Theorem 3.1) that $\mathcal{A}_{v: G L}(\alpha, \beta)$ is inverse closed in $B\left(L^{2}\left(\mathbf{R}^{d}\right)\right)$. The algebra $\mathcal{A}_{v ; G L}$ is naturally associated to the twisted convolution algebra $\left(l_{v}^{1}\left(\mathbf{Z}^{2 d}\right), \sharp_{\theta}\right)$, where

$$
\left(\mathbf{a} \sharp_{\theta} \mathbf{b}\right)(m, n)=\sum_{k, l \in \mathbf{Z}^{d}} a_{k l} b_{m-k, n-l} \cdot e^{2 \pi i \theta(m-k) \cdot l} .
$$

In this setting, the above result is equivalent to saying (Theorem 2.14 in 12]) that for any $\mathbf{a} \in l_{v}^{1}\left(\mathbf{Z}^{2 d}\right)$ so that the convolution operator $L_{\mathbf{a}}: l^{2}\left(\mathbf{Z}^{2 d}\right) \rightarrow l^{2}\left(\mathbf{Z}^{2 d}\right)$, $\mathbf{x} \mapsto L_{\mathbf{a}}(\mathbf{x})=\mathbf{a}_{\theta} \mathbf{x}$, is invertible in $B\left(l^{2}\left(\mathbf{Z}^{2 d}\right)\right)$, then its inverse is of the form $L_{\mathbf{a}}^{-1}=L_{\mathbf{b}}$ for some $\mathbf{b} \in l_{v}^{1}\left(\mathbf{Z}^{2 d}\right)$. The proof of this result relies heavily on abstract results on group algebras of locally compact nilpotent groups ([21, [17]). As the authors point out, analyzing spectral properties of group algebras is not usually an easy business. 
Again in the context of time-frequency analysis, Gröchenig in [11] translated a result by J. Sjöstrand [28] using the modulation space $M_{v}^{\infty, 1}$. The operator algebra

$$
\begin{aligned}
\mathcal{S}_{v}=\left\{T \in B\left(L^{2}\left(\mathbf{R}^{d}\right)\right) \mid T\right. & =\int_{\mathbf{R}^{2 d}} d \lambda \sigma(\lambda) U_{\lambda}, \\
\|T\|_{\mathcal{S}_{v}} & \left.:=\|\sigma\|_{M_{v}^{\infty, 1}}:=\int_{\mathbf{R}^{d}} d q \sup _{p \in \mathbf{R}^{d}}\left|V_{\gamma} \sigma(q, p)\right| v(q, p)\right\}
\end{aligned}
$$

is shown to be inverse closed in $B\left(L^{2}\left(\mathbf{R}^{d}\right)\right)$.

Another example of an inverse closed algebra is furnished by the Baskakov class of matrices that have some off-diagonal decay. In [5] Baskakov proves the Banach algebra:

$$
B_{v}=\left\{A=\left(A_{m, n}\right)_{m, n \in \mathbf{Z}^{d}} \in B\left(l^{2}\left(\mathbf{Z}^{d}\right)\right)\left|\|A\|_{B_{v}}:=\sum_{k \in \mathbf{Z}^{d}} v(k) \sup _{m \in \mathbf{Z}^{d}}\right| A_{m, m-k} \mid<\infty\right\}
$$

with $v$ a subexponential weight that is inverse closed and also obtains estimates of the entries of the inverse matrix. The unweighted version of this result have been proved in [8] (30]). These results have also been obtained independently by Gröchening and Leinert in [13 using a Banach algebra technique. In [3] we used Baskakov's result to establish localization results for Gabor-like frames.

In this paper we extend previously known results to the Banach algebra (4) and its weighted version (14). Besides the intrinsic interest of a new Wiener type lemma, we are motivated by two problems in time-frequency analysis. One problem concerns the Heil-Ramanathan-Topiwala (HRT) conjecture, and the other problem relates to the time-frequency analysis of communication channels.

The HRT conjecture (see [14]) states that finitely many distinct time-frequency shifts of one $L^{2}$ function are linearly independent (over $\mathbf{C}$ ). This means, for any finite subset of $\mathbf{R}^{2 d}, \Lambda \subset \mathbf{R}^{2 d}$, and $g \in L^{2}\left(\mathbf{R}^{d}\right)$,

$$
\sum_{\lambda \in \Lambda} c_{\lambda} U_{\lambda} g=0 \Rightarrow c_{\lambda}=0, \forall \lambda \in \Lambda
$$

When $\Lambda$ is a subset of a lattice, the claim was positively proved by Linnell in [19]; however the general case, as far as we know, is still open. The problem can be recast into a spectral analysis problem. More specifically the HRT conjecture is equivalent to proving that for any finite subset $\Lambda \subset \mathbf{R}^{2 d}$ and complex numbers $\left(c_{\lambda}\right)_{\lambda \in \Lambda}$, the bounded operator $T=\sum_{\lambda \in \Lambda} c_{\lambda} U_{\lambda}$ has no pure point spectrum. Motivated by this problem, one is naturally led to an algebra of type (4). Our result in this paper (Theorem 3.10) is one step toward analyzing spectral properties of such operators. Here we prove that any $T$ in $\mathcal{A}$ (in particular this works for finite linear combinations of time-frequency shifts) cannot have isolated eigenvalues of finite multiplicity. For our setting, this is the best result one can hope to obtain (see Remark 3.11).

In communication theory, a multipath time-varying communication channel is modeled as a linear superposition of time-frequency shifts (see [29]). Often the channel model contains finitely many time-frequency shifts, or infinitely many but fast-decaying coefficients, so naturally, the channel transfer operator is in algebra $\mathcal{A}_{v}$. One problem is channel equalization (or deconvolution) by which one has to invert the channel transfer operator. Assuming this operator is invertible on the space of finite energy signals, then our result says the inverse is also a superposition of time-frequency-shifts, with absolutely summable coefficients. The coefficient 
decay rate gives the convergence rate of finite approximation methods. In this context our results (Theorems 3.6, 3.12) give estimates of this decay. We also obtain necessary and sufficient conditions for operators in $\mathcal{A}$ to have bounded support (Theorem 3.14).

Another contribution of this paper is the explicit construction of a faithful tracial state on $\mathcal{A}$ that yields several consequences. In particular we show that $\mathcal{A}$ does not contain any compact operator, from where we obtain as a corollary the partial answer to the HRT conjecture. We also prove Paley-Wiener type extensions for this algebra.

Throughout this paper we use the following notation. For a set $I,|I|$ denotes the cardinal of set $I$ (i.e. the number of points contained in $I$ ); for $x \in \mathbf{R}^{d},|x|$ denotes its max-norm $\left(l^{\infty}\right)$, whereas $\|x\|$ denotes the Euclidian $\left(l^{2}\right)$ norm; $B_{r}(x)$ denotes the closed ball of radius $r$ centered at $x$ with respect to norm $|\cdot|, B_{r}(x)=$ $\left\{y \in \mathbf{R}^{d}|| x-y \mid \leq r\right\}$. Thus $\left\{B_{1}(n) ; n \in \mathbf{Z}^{d}\right\}$ forms a covering (but not disjoint) partition of $\mathbf{R}^{d} ; E_{r}(x)$ denotes the Euclidian closed ball of radius $r$ centered at $x$, $E_{r}(x)=\left\{y \in \mathbf{R}^{d} \mid\|x-y\| \leq r\right\} ; \mathcal{F}$ denotes the unitary Fourier transform with the following normalization:

$$
f \mapsto \mathcal{F} f(\omega)=\frac{1}{(2 \pi)^{d / 2}} \int_{\mathbf{R}^{d}} e^{-i \omega x} f(x) d x .
$$

We will frequently use $\lambda, \mu$ to denote time-frequency points in $\mathbf{R}^{2 d}$, e.g. $\lambda=$ $(t, \omega)$ of components $t, \omega \in \mathbf{R}^{d}$. For a weight $v$ we denote by $l_{v}^{1}\left(\mathbf{R}^{n}\right)$ (or just $l_{v}^{1}$ when there is no danger of confusion) the space of functions $c: \mathbf{R}^{n} \rightarrow \mathbf{C}$ so that $\|c\|_{l_{v}^{1}}:=\sum_{x \in \mathbf{R}^{n}} v(x)|c(x)|<\infty$. For $0<p<\infty$ we let $l^{p}\left(\mathbf{R}^{n}\right)$ (or merely $l^{p}$, when no danger of confusion) denote the space of functions $c: \mathbf{R}^{n} \rightarrow \mathbf{C}$ so that $\|c\|_{p}:=\left(\sum_{x \in \mathbf{R}^{n}}|c(x)|^{p}\right)^{1 / p}<\infty$. We will frequently use the notation $c_{x}=c(x)$. The support of $c$ is defined by $\operatorname{supp}(c)=\left\{\lambda \in \mathbf{R}^{n} \mid c_{\lambda} \neq 0\right\}$, and for any $c$ in $l^{p}$ with $0<p<\infty$ or $l_{v}^{1}$ it is always a finite or countable subset of $\mathbf{R}^{n}$. For $p=\infty, l^{\infty}$ represents the set of bounded functions on $\mathbf{R}^{n}$, not necessarily of finite or countable support, and $\|c\|_{\infty}=\sup _{x}\left|c_{x}\right|$. For any $p \geq 1, l^{p}$ is a Banach space with $\|\cdot\|_{p}$ norm. Note $l^{p}\left(\mathbf{R}^{n}\right)$ is not separable for any $p$. In particular $l^{2}\left(\mathbf{R}^{n}\right)$ does not have a countable orthonormal basis. $l^{p}\left(\mathbf{R}^{n}\right)$ and $l_{v}^{1}\left(\mathbf{R}^{n}\right)$ are the corresponding $L^{p}$ and $L_{v}^{1}$ spaces for $\mathbf{R}^{n}$ endowed with discrete topology.

The organization of this paper is the following. Section 2 introduces weighted algebras constructions, and Section 3 contains our main results. Section 4 contains comments on different approaches in prior literature, and Section 5 contains proofs of these results.

\section{Weighted time-FREQUENCY BANACH ALGEBRAS}

In this paper a weight $v$ is a nonnegative and radially nondecreasing function on $\mathbf{R}^{d}$ so that $v(0)=1$ and $v(-x)=v(x)$. Let $w: \mathbf{R}^{+} \rightarrow \mathbf{R}^{+}$be the function $w(r)=\max _{\|x\|=r} v(x)$. We define the following (see also [13]):

(a) The weight $v$ is said to be submultiplicative if it satisfies

$$
v(x+y) \leq v(x) v(y) .
$$

(b) The weight $v$ is said to satisfy the GRS (Gelfand-Raikov-Shilov) condition if

$$
\lim _{n \rightarrow \infty}(w(n r))^{1 / n}=1, \forall r \geq 0 .
$$


(c) The weight $v$ is called admissible if it is submultiplicative and satisfies the GRS condition.

Example 2.1 (13]). Typical examples of admissible weights are the polynomial weights, $v(x)=(1+\|x\|)^{s}$ for some $s \geq 0$, and the subexponential weights, $v(x)=$ $e^{\alpha\|x\|^{\beta}}$, for some $\alpha \geq 0$ and $0<\beta<1$. More generally, the following is also an admissible weight (see [13]), $v(x)=e^{\alpha\|x\|^{\beta}}(1+\|x\|)^{s} \log ^{t}(e+\|x\|)$, where $\alpha, s, t \geq 0$, $0<\beta<1$.

Note the exponential weight $v(x)=e^{\alpha\|x\|}$ with $\alpha>0$ is not admissible. It is submultiplicative, but does not satisfy the GRS condition.

Throughout this paper all the weights are assumed to be at least submultiplicative. Except for Lemma 5.2 and the proof of Theorem 3.12, all weights considered in the rest of the paper are admissible. In Lemma 5.2 we consider the less restrictive submultiplicative weights to cover the case of exponential weights needed in the proof of Theorem 3.12 ,

For a weight $v$, we let $\mathcal{A}_{v}$ denote the algebra of time-frequency operators with $l_{v}^{1}$ summable coefficients,

$$
\mathcal{A}_{v}=\left\{T=\sum_{\lambda} c_{\lambda} U_{\lambda} ;\|T\|_{\mathcal{A}_{v}}:=\sum_{\lambda} v(\lambda)\left|c_{\lambda}\right|<\infty\right\} .
$$

This is a subalgebra of the bounded operators $B\left(L^{2}\left(\mathbf{R}^{d}\right)\right)$. Furthermore, for every $T \in \mathcal{A}_{v},\|T\|_{B\left(L^{2}\left(\mathbf{R}^{d}\right)\right)} \leq\|T\|_{\mathcal{A}_{v}}$. Thus the $C^{*}$ algebra $\mathbf{A}$ obtained by closing any one of $\mathcal{A}_{v}$ with respect to the operator norm $\|\cdot\|_{B\left(L^{2}\left(\mathbf{R}^{d}\right)\right)}$ includes $\mathcal{A}$ and hence every $\mathcal{A}_{v}$.

For two Banach algebras $A$ and $B$, we call $A$ an inverse closed algebra in $B$, if any element $x \in A$ that is invertible in $B$ is invertible in $A, x^{-1} \in A$. Neimark in 22. calls $(A, B)$ a Wiener pair, whereas Baskakov in [5] calls $A$ a full algebra in $B$.

\section{MAin Results}

In the following $v$ denotes an admissible weight. In particular $v$ can be the constant function $v=1$ (the unweighted case).

Theorem 3.1 (Spectral invariance). Assume $T=\sum_{\lambda} c_{\lambda} U_{\lambda} \in \mathcal{A}_{v}$. Then the spectral radii with respect to algebras $B\left(L^{2}\left(\mathbf{R}^{d}\right)\right)$ and $\mathcal{A}_{v}$ are equal to one another,

$$
r_{B\left(L^{2}\left(\mathbf{R}^{d}\right)\right)}(T)=r_{\mathcal{A}_{v}}(T) \text {. }
$$

Theorem 3.2 (Wiener lemma for TF operators). The algebra $\mathcal{A}_{v}$ is inverse closed in $B\left(L^{2}\left(\mathbf{R}^{d}\right)\right)$. Explicitly this means, if $T=\sum_{\lambda \in \Lambda} c_{\lambda} U_{\lambda}$ for some $\mathbf{c} \in l^{1}\left(\mathbf{R}^{2 d}\right)$ with $\Lambda=\operatorname{supp}(\mathbf{c})$, and $T$ is invertible in $B\left(L^{2}\left(\mathbf{R}^{d}\right)\right)$, then there is $\mathbf{d} \in l^{1}\left(\mathbf{R}^{2 d}\right)$ with $\Sigma=\operatorname{supp}(\mathbf{d})$ so that $T^{-1}=\sum_{\sigma \in \Sigma} d_{\sigma} U_{\sigma}$.

Immediate corollaries of this result are the following:

Corollary 3.3. For any $T \in \mathcal{A}_{v}$ its spectrum with respect to the algebra $\mathcal{A}_{v}$ coincides to the spectrum with respect to the algebra $B\left(L^{2}\left(\mathbf{R}^{d}\right)\right)$. Explicitly this means

$$
s p_{B\left(L^{2}\left(\mathbf{R}^{d}\right)\right)}(T)=s p_{\mathcal{A}_{v}}(T)
$$


Corollary 3.4. Assume $T=\sum_{\lambda \in \mathbf{R}^{2 d}} c_{\lambda} U_{\lambda}$ with $\sum_{\lambda}\left|c_{\lambda}\right|<\infty$ invertible in $B\left(L^{2}\left(\mathbf{R}^{d}\right)\right)$. Then $T$ is invertible in all $B\left(L^{p}\left(\mathbf{R}^{d}\right)\right)$, with $0<p \leq \infty$.

Corollary 3.5. Let $T=\sum_{t \in \mathbf{R}^{d}} m_{t} S_{t}$ be a bounded invertible operator on $L^{2}\left(\mathbf{R}^{d}\right)$ so that $\sum_{t}\left\|m_{t}\right\|_{A P}<\infty$. Then $T^{-1}=\sum_{t \in \mathbf{R}^{d}} n_{t} S_{t}$ with $n_{t} \in A P$ so that $\sum_{t}\left\|n_{t}\right\|_{A P}<$ $\infty$.

The following theorem gives an explicit estimate of the $\mathcal{A}_{v}$ norm of the inverse when the operator has finite support.

Theorem 3.6 (Norm of the inverse). Assume $T=\sum_{\lambda \in \Lambda} c_{\lambda} U_{\lambda}$ with $|\Lambda|=N<\infty$ and $R_{0}=\max _{\lambda \in \Lambda}\|\lambda\|$. Assume $T$ is invertible in $B\left(L^{2}\left(\mathbf{R}^{d}\right)\right)$, and hence in $\mathcal{A}_{v}$ as well (by Theorem 3.2). Denote $A=\left\|T^{-1}\right\|_{B\left(L^{2}\left(\mathbf{R}^{d}\right)\right)}^{2}, B=\|T\|_{B\left(L^{2}\left(\mathbf{R}^{d}\right)\right)}^{2}$, and $\rho=\max \left(1,2 R_{0}\right)$, and assume a polynomial weight $w(x)=C(1+x)^{m}$ for some $C>0$ and $m \in \mathbf{N}$. Then

$$
\left\|T^{-1}\right\|_{\mathcal{A}_{v}} \leq \frac{C \rho^{m}\|T\|_{\mathcal{A}_{v}}}{A}(m+N) !\left(\frac{A+B}{2 A}\right)^{m+N} .
$$

Now consider $\mathcal{G}=\left\{g_{m, n ; \alpha, \beta}:=U_{\beta n, 2 \pi \alpha m} g \mid m, n \in \mathbf{Z}^{d}\right\}$ a Gabor frame for $L^{2}\left(\mathbf{R}^{d}\right)$, with $\alpha, \beta>0, \alpha \beta \leq 1$, and a dual Gabor frame (not necessarily the canonical dual frame) $\tilde{\mathcal{G}}=\left\{\tilde{g}_{m, n ; \alpha, \beta}:=U_{\beta n, 2 \pi \alpha m} \tilde{g} \mid m, n \in \mathbf{Z}^{d}\right\}$. For details on Gabor frame theory we refer the reader to e.g. [9]. The following theorem gives an explicit construction of the faithful tracial state:

$$
\gamma: \mathcal{A} \rightarrow \mathbf{C}, \gamma\left(\sum_{\lambda} c_{\lambda} U_{\lambda}\right)=c_{0}
$$

This trace extends to $\mathbf{A}$, the completion of $\mathcal{A}$ with respect to the operator norm, which is a $C^{*}$ algebra.

Theorem 3.7 (Trace on $\mathbf{A}$ ). For any $T \in \mathbf{A}$,

(19) $\gamma(T)=\frac{1}{(\alpha \beta)^{d}} \lim _{M, N \rightarrow \infty} \frac{1}{(2 M+1)^{d}(2 N+1)^{d}} \sum_{|m| \leq M} \sum_{|n| \leq N}\left\langle T g_{m, n ; \alpha, \beta}, \tilde{g}_{m, n ; \alpha, \beta}\right\rangle$

is the faithful tracial state (18) on $\mathbf{A}$, independent of the choice of the Gabor frame $\mathcal{G}$.

Next we have

Corollary 3.8. For any operator $T=\sum_{\lambda} c_{\lambda} U_{\lambda} \in \mathcal{A}$

$$
\left|c_{\lambda}\right| \leq\|c\|_{\infty} \leq\|c\|_{2} \leq\|T\|_{B\left(L^{2}\left(\mathbf{R}^{d}\right)\right)} \leq\|c\|_{1}
$$

and

$$
\begin{aligned}
c_{\lambda} & =\gamma\left(U_{\lambda}^{*} T\right) \\
& =\lim _{M, N \rightarrow \infty} \frac{1}{(\alpha \beta)^{d}} \frac{1}{(2 M+1)^{d}(2 N+1)^{d}} \sum_{|m| \leq M} \sum_{|n| \leq N}\left\langle U_{\lambda}^{*} T g_{m, n ; \alpha, \beta}, \tilde{g}_{m, n ; \alpha, \beta}\right\rangle .
\end{aligned}
$$


As a corollary of this result we obtain that $\mathbf{A}$ (and hence $\mathcal{A}$ as well) cannot contain compact operators:

Corollary 3.9. Assume $T \in \mathbf{A}$ is a compact operator. Then $T=0$.

Since for any finite set $\Lambda \in \mathbf{R}^{2 d}$ and complex scalars $\left(c_{\lambda}\right)$ the operator $T=$ $\sum_{\lambda \in \Lambda} c_{\lambda} U_{\lambda}$ is in $\mathcal{A}$, we obtain the following theorem that gives a partial answer to the HRT conjecture:

Theorem 3.10. Any operator $T \in \mathcal{A}$ (in particular, any finite linear combination of time-frequency shifts) cannot have an isolated eigenvalue of finite multiplicity. Hence the pure point spectrum, if it is nonempty, can only contain eigenvalues of infinite multiplicity, or eigenvalues that belong to the continuous part of the spectrum as well.

Remark 3.11. This theorem is optimal with respect to algebra $\mathcal{A}$. This means that there are operators in $\mathcal{A}$ that have isolated eigenvalues but of infinite multiplicity. Indeed, consider the Gaussian window $\gamma(x)=\exp \left(-x^{2} / 2\right)$ and the Riesz basic sequence it generates for some parameters $\alpha, \beta>1, \Gamma=\left\{\gamma_{m, n ; \alpha, \beta} ; m, n \in \mathbf{Z}^{d}\right\}$. Let $\tilde{\gamma}$ be the canonical dual window. Note that both $\gamma, \tilde{\gamma} \in M^{1}$ (by [12]). Now consider the orthogonal projection $P$ onto the span of $\Gamma$. It is immediate to prove that $P$ commutes with all time-frequency shifts $U_{n \beta, 2 \pi m \alpha}$. Hence $P$ belongs to the commutant $\left\{U_{b e, 0}, U_{0,2 \pi \alpha}, I\right\}^{\prime}$ which is the von Neumann algebra generated by $U_{n / \alpha, 2 \pi m / \beta}$. The decomposition of $P$ with respect to these unitary generators is given by the Wexler-Raz formula

$$
P=\sum_{m, n \in \mathbf{Z}^{d}} c_{n, m} U_{\frac{n}{\alpha}, \frac{2 \pi m}{\beta}}
$$

with $c_{n, m}=\frac{1}{(\alpha \beta)^{d}}\left\langle\gamma_{n, m ; \frac{1}{\alpha}, \frac{2 \pi}{\beta}}, \tilde{\gamma}\right\rangle$. The coefficients are absolutely summable, which implies $P \in \mathcal{A}$. But clearly the spectrum of $P$ consists of only two elements, 0 and 1 , each of infinite multiplicity, since otherwise this would contradict Theorem 3.10 . The multiplicity of eigenvalue 0 represents the deficit of $\Gamma$, defined as the smallest cardinal of a set of vectors so that its union with $\Gamma$ becomes a Riesz basis for $L^{2}\left(\mathbf{R}^{d}\right)$. This is the dual problem to the excess problem we analyzed in [1, 2, 3, 4. Thus the deficit of any Gabor Riesz basic sequence with generator in $M^{1}$ can only be infinite (zero is ruled out by the Balian-Law no-go result).

For finite support operators as above, we can estimate the decay rate of the coefficients of the inverse operator. In general such operators are in $\mathcal{A}_{v}$ for any subexponential weight $v$. Hence the inverse operator would have coefficients that are summable with respect to the same weight. Even more can be said:

Theorem 3.12. Let $T=\sum_{\lambda \in \Lambda} c_{\lambda} U_{\lambda}$ be an invertible operator with $\Lambda \subset \mathbf{R}^{2 d}$ a finite set. Then there is $\delta>0$ so that if $T^{-1}=\sum_{\mu \in \mathbf{R}^{2 d}} d_{\mu} U_{\mu}$, then

$$
\sum_{\mu \in \mathbf{R}^{2 d}} e^{\delta|\mu|}\left|d_{\mu}\right|<\infty .
$$

This result generalizes the classic statement (see e.g. [31]) that the reciprocal of a trigonometric polynomial that does not vanish on the unit circle has exponentially decaying Fourier coefficients. Our result is stronger than just simply saying the 
coefficients of the inverse operator should decay exponentially fast. It also controls the "tail" of the summation. To make this point more precise, we notice that (22) is equivalent to a Wiener amalgam type norm:

$$
\sum_{n \in \mathbf{Z}^{2 d}} e^{\delta|n|} \sum_{\mu \in B_{1}(n)}\left|d_{\mu}\right|<\infty
$$

where $B_{1}(n)$ is the ball of radius 1 centered at $n, B_{1}(n)=\left\{\lambda \in \mathbf{R}^{2 d}|| n-\lambda \mid \leq 1\right\}$. Hence there is a constant $C>0$ so that for all $R>0$,

$$
\sum_{\mu \in \mathbf{R}^{2 d},|\mu| \geq R}\left|d_{\mu}\right|<C e^{-\delta R} .
$$

Another equivalent statement to Theorem 3.12 is given by Corollary 3.13 below. First we need to introduce a Banach space. Let us denote by $L^{2, \infty}\left(\mathbf{R}^{d} \times I\right)$ the mixed norm Banach space

$$
L^{2, \infty}\left(\mathbf{R}^{d} \times I\right)=\left\{f: \mathbf{R}^{d} \times I \rightarrow \mathbf{C} ;\|f\|_{2, \infty}^{2}:=\sup _{y \in I} \int_{\mathbf{R}^{d}}|f(x, y)|^{2} d x<\infty\right\}
$$

where $I \subset \mathbf{R}^{d}$ is a compact neighborhood of the origin. Then the unitary $U_{\lambda}$ extends from $L^{2}\left(\mathbf{R}^{d}\right)$ to $L^{2, \infty}\left(\mathbf{R}^{d} \times I\right)$ simply by:

$$
\mathbf{U}_{\lambda} f(x, y)=e^{i\langle\omega, x+i y\rangle} f(x-t, y), \lambda=(t, \omega),(x, y) \in \mathbf{R}^{d} \times I .
$$

An operator $T=\sum_{\lambda} c_{\lambda} U_{\lambda}$ extends to $\mathbf{T}=\sum_{\lambda} c_{\lambda} \mathbf{U}_{\lambda}$ under some conditions. Clearly all finite or compactly supported operators of $\mathcal{A}$ can be extended to $L^{2, \infty}\left(\mathbf{R}^{d} \times I\right)$. Theorem 3.14 gives necessary and sufficient conditions for such an extension to exist.

Corollary 3.13. Let $T=\sum_{\lambda \in \Lambda} c_{\lambda} U_{\lambda}$ be a finitely supported invertible operator in $\mathcal{A}$. Then for some compact neighborhood I of the origin whose size depends on the operator $T$, the inverse $T^{-1}=\sum_{\mu} d_{\mu} U_{\mu}$ extends to $L^{2, \infty}\left(\mathbf{R}^{d} \times I\right)$ to the inverse of the extension, that is, to:

$$
\mathbf{T}^{-1}=\sum_{\mu} d_{\mu} \mathbf{U}_{\mu}
$$

An alternative statement is that extension and inversion operations commute for some compact neighborhood of the origin.

Theorem 3.14. (1) Assume $T=\sum_{\lambda \in \Lambda} c_{\lambda} U_{\lambda} \in \mathcal{A}$ so that $\Lambda \subset \mathbf{R}^{d} \times E_{\Omega}(0)$ for some $\Omega>0$, where $E_{\Omega}(0)=\left\{\omega \in \mathbf{R}^{d} \mid\|\omega\| \leq \Omega\right\}$. Then for any $\rho>0$, $T$ extends to $X_{\rho}=L^{2, \infty}\left(\mathbf{R}^{d} \times E_{\rho}(0)\right)$ with operator norm bounded by:

$$
\|\mathbf{T}\|_{B\left(X_{\rho}\right)} \leq C e^{\rho \Omega}
$$

for $C=\|T\|_{\mathcal{A}}$.

(2) Conversely, assume $T=\sum_{\lambda \in \Lambda} c_{\lambda} U_{\lambda} \in \mathcal{A}$ can be extended to $X_{\rho}$ for all $\rho>0$, with a norm bounded as in (28), for some $C>0$ and $\Omega>0$ independent of $\rho$. Then $\operatorname{supp}(T) \subset \mathbf{R}^{d} \times E_{\Omega}(0)$.

(3) Assume $T=\sum_{\lambda \in \Lambda} c_{\lambda} U_{\lambda} \in \mathcal{A}$ so that $\Lambda \subset E_{D}(0) \times \mathbf{R}^{d}$ for some $D>0$. Then for any $\rho>0$, the operator $S=\mathcal{F}^{*} T \mathcal{F}$, where $\mathcal{F}$ denotes the Fourier 
transform, extends to $X_{\rho}$ with operator norm bounded by

$$
\|\mathbf{S}\|_{B\left(X_{\rho}\right)} \leq C e^{\rho D}
$$

for $C=\|T\|_{\mathcal{A}}$.

(4) Conversely, assume the Fourier conjugate $\mathcal{F}^{*} T \mathcal{F}$ of $T=\sum_{\lambda \in \Lambda} c_{\lambda} U_{\lambda} \in \mathcal{A}$ can be extended to $X_{\rho}$ for all $\rho>0$ with a norm bounded as in (29) with $C$ and $D$ independent of $\rho$. Then $\operatorname{supp}(T) \subset E_{D}(0) \times \mathbf{R}^{d}$.

\section{Connections to prior literature}

In this section we discuss the two ingredients developed in this paper: Wiener lemma type results, and the faithful tracial state in Gabor analysis. For each of these results we discuss prior results and approaches presented in literature, and the strengths and shortcomings of each method. For precise definitions and more details of the results we refer the reader to the corresponding paper.

4.1. Discussion about the Wiener lemma and alternate proofs. The closest paper to our analysis is [12] by Gröchenig and Leinert. The authors proved the analogous statement to Theorem 3.2 but only for the lattice case, that is, the algebra of time-frequency shifts from a lattice. As an off-shot of this approach, the authors obtained a very nice localization result regarding dual Gabor frame generators. More specifically, if $\left\{g_{m, n ; \alpha, \beta} ; m, n \in \mathbf{Z}^{d}\right\}$ is a Gabor frame for $L^{2}\left(\mathbf{R}^{d}\right)$ with $g \in M_{v}^{1}$, the modulation space associated to an admissible weight $v$, then the canonical dual frame $\left\{\tilde{g}_{m, n ; \alpha, \beta} ; m, n \in \mathbf{Z}^{d}\right\}$ has the generator $\tilde{g} \in M_{v}^{1}$. One may ask whether the methods used in that paper hold in our case. The answer is affirmative. Indeed, the main tool used in [12] is the fact that the Banach algebra $l_{v}^{1}\left(\mathbf{Z}^{2 d}\right)$, with the twisted convolution $\sharp$, is symmetric, which in turn is a consequence of Hulanicki's and Ludwig's results on symmetric group algebras associated to nilpotent groups. The very same result applies to our case where the discrete countable group $\mathbf{Z}^{2 d}$ is simply replaced by $\mathbf{R}^{2 d}$ with discrete topology. Then, as mentioned in the Introduction, Theorems 3.2 and 3.1 become consequences of Hulanicki's theorem from e.g. [16]. However, by invoking a general abstract result one does not obtain the norm estimates of Theorem 3.7 nor the localization results of Theorems 3.12 and 3.14. We preferred to present an explicit and self-contained proof of Theorem 3.2 for two reasons: (i) for the benefit of reader unfamiliar with the symmetry property of nilpotent group algebras; and (ii) to obtain explicit estimates of the inverse operator coefficients.

We mentioned earlier an important consequence contained in [12], namely the localization result of the canonical dual Gabor frame generator. One may ask if there is an analogous consequence to our more general case. The most natural guess would be to analyze the canonical dual of irregular Gabor frames. Unfortunately, unlike the regular (i.e. lattice) case, the frame operator may not necessarily belong to $\mathcal{A}_{v}$ and hence no conclusion can be drawn from our analysis. Fortunately one can use another approach to recover the results of [12] and prove the localization result in the irregular case. This alternative approach is used in [10, 7], and independently in [3, 4, together with Baskakov's result mentioned earlier in the Introduction, or other similar variations (e.g. Sjöstrand's lemma in [28]). Indeed, the frame operator of an irregular Gabor frame with generator in $M^{1}$ has a matrix representation with respect to a "nice" Gabor frame that is dominated by a Toeplitz matrix with $l^{1}$ generating sequence. In [3] such frames are called $l^{1}$ self-localized frames. 
The associated matrix of such frames admits a pseudoinverse, because of frame condition. Using the Baskakov Theorem and holomorphic functional calculus one obtains that the pseudoinverse has the same off-diagonal decaying property which proves the localization result for the canonical dual frame. In the regular case, the inverse of an invertible operator that is a linear combination of time-frequency shifts from a lattice is also a linear combination of time-frequency shifts on the same lattice. (Here we use "linear combination" to denote the generators of a $C^{*}$ algebra, hence convergence in operator norm.) Thus distinct time-frequency labels associated to the inverse operator are always well separated. This fact combined with Baskakov's result applied to the pseudoinverse matrix gives an alternative proof to the case considered in [12] (see also [13]).

The irregular case is fundamentally different from the lattice case. It is true that an operator in $\mathcal{A}$ always has support contained into a countable generated discrete group of the time-frequency plane. However the main obstruction in the irregular case is the fact that the time-frequency labels of the inverse of an operator $T \in \mathcal{A}$ are not necessarily well-separated, even when $T$ has finite support. Indeed, if $T=\sum_{\lambda \Lambda} c_{\lambda} U_{\lambda}$ with $|\Lambda|<\infty$ is invertible in $B\left(L^{2}\left(\mathbf{R}^{d}\right)\right)$, then $T^{-1}=\sum_{\mu} d_{\mu} U_{\lambda}$ with convergence in operator norm in $\mathbf{A}$. However, in general, $\operatorname{supp}\left(T^{-1}\right)$ has accumulation points in $\mathbf{R}^{d}$. This fact makes difficult the application of Baskakov's Theorem to irregular frames. To better understand this obstruction, we only remark here that the conclusion that can be drawn along this line of reasoning is the following statement. If $T=\sum_{\lambda} c_{\lambda} U_{\lambda} \in \mathcal{A}$ is invertible in $B\left(L^{2}\left(\mathbf{R}^{d}\right)\right)$, then its inverse $T^{-1}=\sum_{\mu} d_{\mu} U_{\mu}$ satisfies

$$
\sum_{k \in \mathbf{Z}^{2 d}} \sup _{\mu \in B_{1}(k)}\left|d_{\mu}\right|<\infty .
$$

Clearly this statement is weaker than Theorem 3.2 that claims $\sum_{\mu}\left|d_{\mu}\right|<\infty$.

4.2. Faithful tracial states in Gabor analysis. In [6] Daubechies, Landau, and Landau present an explicit formula for the faithful tracial state on the $W^{*}$ algebra $\mathbf{W}_{a, b}$ generated by $\left\{M_{2 \pi m a} T_{n b} ; m, n \in \mathbf{Z}\right\}$. They showed that $\mathbf{W}_{a, b}$ is a $I I_{1}$ factor when $a b \notin \mathbf{Q}$ (the result is also known from the rotation algebra theory, see e.g. [24]), that has a unique faithful tracial state. In general, regardless of rationality of $a b$, a faithful tracial state is defined as the coefficient $c_{0,0}$ of its strongly convergent uniquely defined decomposition $T=\sum_{m, n} c_{m, n} U_{n b, 2 \pi m a}$. They showed that this number $\left(c_{0,0}\right)$ is computable using the formula

$$
c_{0,0}=\frac{1}{a b} \sum_{k=0}^{J}\left\langle T 1_{I_{k}}, 1_{I_{k}}\right\rangle
$$

where $J$ is the largest integer smaller than or equal to $a b$, and the $J+1$ intervals $I_{0}, I_{1}, \ldots, I_{J-1}, I_{J}$ are given by $\left[0, \frac{1}{a}\right],\left[\frac{1}{a}, \frac{2}{a}\right], \ldots,\left[\frac{J-1}{a}, \frac{J}{a}\right],\left[\frac{J}{a}, b\right]$.

In this paper we extend this tracial state from the algebra generated by timefrequency shifts on a lattice to the algebra generated by all time-frequency shifts. Note however the following limitation of our method. In [6] the faithful tracial state applies to a $W^{*}$ algebra, whereas our Theorem 3.7 applies only to a $C^{*}$ algebra. The tracial state $\gamma$ of (18) cannot be extended to the $W^{*}$ algebra generated by $\mathcal{A}$ since this $W^{*}$ algebra is the entire algebra $B\left(L^{2}\left(\mathbf{R}^{d}\right)\right.$ ) (which does not admit a faithful tracial state). Now consider the series of $C^{*}$ algebras $\left(\mathbf{C}_{a, b}\right)_{a, b>0}$ each 
generated respectively by $\left\{U_{n b, 2 \pi m a} ; m, n \in \mathbf{Z}\right\}$ (we restrict ourselves here to the one-dimensional case for convenience of comparison). For any $T \in \mathbf{C}_{a, b}$, for some $a, b>0$, its trace $\gamma(T)$ can be computed either by (31), or by (19). Our formula (19) has the advantage of being independent of lattice parameters $(a, b)$. In particular this shows the tracial states defined by (31) are compatible on operators that belong simultaneously to two different $W^{*}$ algebras (for instance $T \in \mathbf{C}_{a, b} \subset \mathbf{C}_{2 a, b} \cap \mathbf{C}_{a, 2 b}$ ).

We end this section with a comment on Theorem 3.10. In this paper we solve a restricted case of the HRT conjecture, namely we rule out the existence of isolated eigenvalues of finite multiplicity for all finite linear combinations of time-frequency shifts. In fact we obtain this conclusion for any operator of $\mathcal{A}$, hence also for infinite linear combinations of time-frequency shifts with coefficients in $l^{1}$. The other case that was ruled out is the lattice case, that is, when the finitely many time-frequency shifts are from a lattice. This was beautifully proved by Linnel in [19]. One may ask whether the same arguments hold in our more general case. There is difficulty in trying to do so, namely $\gamma$ is a faithful tracial state on a $C^{*}$ algebra (in this paper) unlike the $W^{*}$ algebra $\mathbf{W}_{a, b}$ considered in [19]. This difference prevents us from having a similar proof in our setting. We currently study ways to bypass this difficulty.

\section{Proof of Results}

The order of proofs is the following. First we prove the spectral invariance Theorem 3.1, from where we derive Theorem 3.2. In the process of proving Theorem 3.1 we obtain the norm estimate (35) used in the proof of Theorem 3.6. Corollaries 3.3. 3.4 and 3.5 follow directly from Theorem 3.2.

In Theorem 3.7 we construct the faithful tracial state on $\mathcal{A}$, and therefore $\mathbf{A}$. This will be proved later in this section. From this result we will derive Corollaries 3.8, 3.9, and Theorem 3.10. Theorems 3.12 and 3.14 will follow after an extension to the Banach space $L^{2, \infty}\left(\mathbf{R}^{d} \times I\right)$.

5.1. Proof of Theorems 3.1, 3.2, 3.6 and Corollaries 3.3, 3.4, 3.5, Theorem 3.1 is obtained in two steps. The first step involves finite linear combinations of time-frequency shifts. In the second step the spectral result is extended to the entire algebra $\mathcal{A}$.

Consider $T=\sum_{\lambda \in \Lambda} c_{\lambda} U_{\lambda}$ with $|\Lambda|<\infty$ a finite linear combination of timefrequency shifts. Note:

$$
T^{n}=\sum_{\sigma \in \Lambda^{(n)}} d_{\sigma} U_{\sigma}
$$

where:

$$
\begin{aligned}
\Lambda^{(n)}= & \Lambda+\Lambda+\cdots+\Lambda=\left\{\lambda_{1}+\cdots+\lambda_{n} \mid \lambda_{1}, \ldots, \lambda_{n} \in \Lambda\right\}, \\
d_{\sigma}= & \sum_{\substack{\lambda_{1}, \ldots, \lambda_{n} \in \Lambda \\
\lambda_{1}+\cdots+\lambda_{n}=\sigma}} c_{\lambda_{1}} \cdots c_{\lambda_{n}} e^{-i t_{1} \omega_{2}} e^{-i t_{2} \omega_{3}} \cdots e^{-i t_{n-1} \omega_{n}},
\end{aligned}
$$

where each $\lambda_{k}=\left(t_{k}, \omega_{k}\right)$. Then by Cauchy-Schwarz,

$$
\left\|T^{n}\right\|_{\mathcal{A}_{v}}=\sum_{\sigma \in \Lambda^{(n)}}\left|d_{\sigma}\right| v(\sigma) \leq\left|\Lambda^{(n)}\right|^{1 / 2} \sup _{\sigma \in \Lambda^{(n)}}|v(\sigma)|\|d\|_{2} .
$$


We estimate next the three factors of the right-hand side and prove the following

Lemma 5.1. Assume $T=\sum_{\lambda \in \Lambda} c_{\lambda} U_{\lambda}$ is an operator in $\mathcal{A}$ with $|\Lambda|<\infty$ and $\Lambda \subset E_{R_{0}}(0)$. Then for any submultiplicative weight $v$, that is, $v(x+y) \leq v(x) v(y)$ for all $x, y$,

$$
\left\|T^{n}\right\|_{\mathcal{A}_{v}} \leq(n+1)^{|\Lambda| / 2} w\left(n R_{0}\right) \cdot\left\|T^{n}\right\|_{B\left(L^{2}\left(\mathbf{R}^{d}\right)\right)}
$$

where $w(a)=\sup _{\|x\|=a} v(x)$.

Proof of Lemma 5.1. An upper bound for the cardinal of set $\Lambda^{(n)}$ in (34) is obtained as follows. Notice that $\lambda_{i}+\lambda_{j}=\lambda_{j}+\lambda_{i}$; therefore any permutation of terms in $\lambda_{1}+\cdots+\lambda_{n}$ would produce the same point $\sigma$. Hence:

$$
\begin{aligned}
\left|\Lambda^{(n)}\right| & \leq\left|\left\{\left(k_{1}, k_{2}, \ldots, k_{|\Lambda|}\right) \mid k_{1}+k_{2}+\cdots+k_{|\Lambda|}=n, k_{1}, k_{2}, \ldots, k_{|\Lambda|} \geq 0\right\}\right| \\
& =\frac{(n+|\Lambda|) \cdots(n+1)}{|\Lambda| !} \leq(n+1)^{|\Lambda|}
\end{aligned}
$$

For the second factor in (34) we need to estimate the radius $R_{n}$ of a ball $E_{R}(0)$ in $\mathbf{R}^{2 d}$ that includes all $\Lambda^{(n)}$. If $R_{0}=\max _{\lambda \in \Lambda}\|\lambda\|$, then for $R_{n}=n R_{0}$ we have $\Lambda^{(n)} \subset E_{R_{n}}(0)$. Since the weight $v$ is radially nondecreasing,

$$
\max _{\sigma \in \Lambda^{(n)}} v(\sigma) \leq w\left(n R_{0}\right) .
$$

The third factor in (34) is a bit more complicated. We need to use the following lemma, which is of intrinsic interest:

Lemma 5.2. For any finite set of time-frequency points $\Sigma=\left\{\sigma_{1}, \ldots, \sigma_{N}\right\}$ there is a function $g \in L^{2}\left(\mathbf{R}^{d}\right)$ so that $\left\{U_{\sigma_{k}} g ; 1 \leq k \leq N\right\}$ is an orthonormal set.

Assume this lemma is proved. Then we apply Lemma 5.2 to the set $\Lambda^{(n)}=$ $\Lambda+\cdots+\Lambda$ and we obtain, on the one hand

$$
\left\|T^{n} g\right\|^{2}=\left\|\sum_{\sigma \in \Lambda^{(n)}} d_{\sigma} U_{\sigma} g\right\|^{2}=\sum_{\sigma \in \Lambda^{(n)}}\left|d_{\sigma}\right|^{2}=\|d\|_{2}^{2}
$$

and on the other hand

$$
\left\|T^{n} g\right\|^{2} \leq\left\|T^{n}\right\|^{2}\|g\|^{2}=\left\|T^{n}\right\|^{2} .
$$

Thus we get:

$$
\|d\|_{2} \leq\left\|T^{n}\right\| .
$$

Putting together (36), (37), (38) into (34) we obtain:

$$
\left\|T^{n}\right\|_{\mathcal{A}_{v}} \leq(n+1)^{|\Lambda| / 2} w\left(n R_{0}\right)\left\|T^{n}\right\|_{B\left(L^{2}\left(\mathbf{R}^{d}\right)\right)}
$$

which proves Lemma 5.1

Remark 5.3. Inequality (38) also follows independently from (20) of Corollary 3.8 .

Taking the $n^{\text {th }}$ root and passing to the limit $n \rightarrow \infty$ we obtain:

$$
r(T)_{\mathcal{A}_{v}} \leq r(T)_{B\left(L^{2}\left(\mathbf{R}^{d}\right)\right)} .
$$

Since the inclusion $\mathcal{A}_{v} \subset B\left(L^{2}\left(\mathbf{R}^{d}\right)\right)$ implies the inverse inclusion of the spectra $s p_{B\left(L^{2}\left(\mathbf{R}^{d}\right)\right)}(T) \subset s p_{\mathcal{A}_{v}}(T)$, one obtains $r_{B\left(L^{2}\left(\mathbf{R}^{d}\right)\right)}(T) \leq r_{\mathcal{A}_{v}}(T)$. This concludes the proof of the spectral radii equation (15) for finite linear combinations of timefrequency shifts. Before going to the second step, we prove Lemma 5.2 
Proof of Lemma 5.2. The statement is equivalent to finding a function $g \in L^{2}\left(\mathbf{R}^{d}\right)$ so that $\left\langle U_{\sigma} g, g\right\rangle=\delta_{\sigma, 0}$, for all $\sigma \in \Delta:=(\Sigma-\Sigma)$. Let $\mathcal{T}$ be the projection of $\Delta$ on the first factor $\mathbf{R}^{d}$, and $\Omega$ be the projection onto the second factor $\mathbf{R}^{d}$. Thus $\Delta \subset \mathcal{T} \times \Omega$. Notice both $\mathcal{T}$ and $\Omega$ are finite sets of points of $\mathbf{R}^{d}$, symmetric about, and containing the origin. Let $\Omega \backslash\{0\}=\left\{\omega_{1}, \ldots, \omega_{M}\right\}$ be an enumeration of $\Omega$, and let $\tau_{\min }, \tau_{\max }>0$ be the radii of two balls around the origin in $\mathbf{R}^{d}$ so that $E_{\tau_{\min }}(0) \cap \mathcal{T}=\{0\}$ and $\mathcal{T} \subset E_{\tau_{\max }}(0)$. We set $g$ as follows:

$$
g=\sqrt{h_{1} * h_{2} * \cdots * h_{M}} /\left\|\sqrt{h_{1} * h_{2} * \cdots * h_{M}}\right\|
$$

where $*$ denotes the usual convolution, and $h_{1}, h_{2}, \ldots, h_{M}$ are constructed as follows. First we construct inductively the sequence $t_{1}, t_{2}, \ldots, t_{M} \in \mathbf{R}^{d}$ so that:

(1) $\left\langle t_{1}, \omega_{1}\right\rangle=\left(2 n_{1}+1\right) \pi$ for some integer $n_{1} \in \mathbf{Z}$ and $\left\|t_{1}\right\|>\tau_{\max }$.

(2) Given $t_{1}, t_{2}, \ldots, t_{k}$ choose $t_{k+1}$ so that: (i) $\left\langle t_{k+1}, \omega_{k+1}\right\rangle=\left(2 n_{k+1}+1\right) \pi$ for some integer $n_{k+1} \in \mathbf{Z}$, and (ii) $\left\|t_{k+1}\right\|>\left\|t_{1}\right\|+\cdots+\left\|t_{k}\right\|+2 M \tau_{\max }$.

With this choice for $\left\{t_{1}, \ldots, t_{M}\right\}$, we set:

$$
h_{k}=1_{E}+1_{t_{k}+E}
$$

where $E=E_{\tau_{\min } / M}(0)$ is the Euclidian ball of radius $\tau_{\min }$ centered at the origin, and $1_{E}, 1_{t_{k}+E}$, are the characteristic functions of $E$, respectively $t_{k}+E$. Note that $g$ is a sum of $2^{M}$ "bump" functions each supported inside balls of radius $\tau_{\min }$ and each at a distance of at least $\tau_{\max }$ from one another. Thus all translates with shifts from $\mathcal{T} \backslash\{0\}$ are disjoint. Hence $\left\langle U_{\mu} g, g\right\rangle=0$ for all $\mu=(t, \omega) \in \Delta$ with $t \in \mathcal{T} \backslash\{0\}$. It only remains to check that $\left\langle M_{\omega_{k}} g, g\right\rangle=0$. Using Fourier transform, this is equivalent to $\mathcal{F}\left(|g|^{2}\right)\left(\omega_{k}\right)=0$. But the choice of $t_{k}$ guarantees that $\mathcal{F}\left(h_{k}\right)\left(\omega_{k}\right)=0$ which concludes the proof of Lemma 5.2.

Now we are ready to go to step 2 of the proof of Theorem 3.1. Now consider $T=\sum_{\lambda} c_{\lambda} U_{\lambda} \in \mathcal{A}_{v}$. Fix $\varepsilon>0$. Let $\Lambda$ be a finite set so that $\sum_{\lambda \in \mathbf{R}^{2 d} \backslash \Lambda}\left|c_{\lambda}\right| v(\lambda)<\varepsilon$. Set $T_{0}=\sum_{\lambda \in \Lambda} c_{\lambda} U_{\lambda}$ and $R=T-T_{0}$. Thus $\|R\| \leq\|R\|_{\mathcal{A}_{v}}<\varepsilon$. For a more convenient notation denote by $s=\left(s_{\sigma}\right)_{\sigma}$ and $r=\left(r_{\rho}\right)_{\rho}$ the coefficients of $T_{0}$, respectively $R$,

$$
s_{\sigma}=\left\{\begin{array}{rll}
c_{\sigma} & \text { if } & \sigma \in \Lambda, \\
0 & & \text { otherwise, }
\end{array} \quad r_{\rho}=\left\{\begin{array}{rll}
0 & \text { if } & \rho \in \Lambda, \\
c_{\rho} & & \text { otherwise }
\end{array}\right.\right.
$$

Now expand $T^{n}=\left(T_{0}+R\right)^{n}$ as follows:

$$
\begin{aligned}
T^{n} & =\sum_{m=0}^{n} \sum_{k, j} R^{k_{1}} T_{0}^{j_{1}} R^{k_{2}} T_{0}^{j_{2}} \cdots R^{k_{l}} T_{0}^{j_{l}} \\
& =\sum_{m=0}^{n} \sum_{k, j} \sum_{\lambda \in \mathbf{R}^{2 d}}\left(r^{k_{1}} \sharp s^{j_{1}} \sharp \cdots \sharp r^{k_{L} \sharp s^{j_{L}}}\right)_{\lambda} U_{\lambda}
\end{aligned}
$$

where $k=\left(k_{1}, k_{2}, \ldots, k_{L}\right), j=\left(j_{1}, j_{2}, \ldots, j_{L}\right)$ are vectors of nonnegative integers so that $k_{1}+\cdots+k_{L}=n-m$ and $j_{1}+\cdots+j_{L}=m$, and $r^{k}=r \sharp \cdots \sharp r$ is the $k$-fold twisted convolution. Then the $\lambda$-coefficient expands into

$$
\begin{aligned}
c_{\lambda}= & \sum_{\rho_{1}, \ldots, \rho_{L} \in \mathbf{R}^{2 d}} \sum_{\sigma_{1}, \ldots, \sigma_{L} \in \mathbf{R}^{2 d}} r_{\rho_{1}}^{k_{1}} e^{-i\left\langle a_{1}, d_{1}-b_{1}\right\rangle} s_{\sigma_{1}-\rho_{1}}^{j_{1}} \\
& \cdot e^{-i\left\langle c_{1}, b_{2}-d_{1}\right\rangle} r_{\rho_{2}-\sigma_{1}}^{k_{2}} e^{-i\left\langle a_{2}, d_{2}-b_{2}\right\rangle} s_{\sigma_{2}-\rho_{2}}^{j_{2}} \ldots \\
& \cdot e^{-i\left\langle c_{L-1}, b_{L}-d_{L-1}\right\rangle} r_{\rho_{L}-\sigma_{L-1}}^{k_{L}} e^{-i\left\langle a_{L}, d_{L}-b_{L}\right\rangle} s_{\sigma_{L}-\rho_{L}}^{j_{L}} \delta_{\sigma_{L}, \lambda}
\end{aligned}
$$


where $\rho_{l}=\left(a_{l}, b_{l}\right)$ and $\sigma_{l}=\left(c_{l}, d_{l}\right)$ are the components of the $2 L$ phase-space points constrained by $\sigma_{L}=\lambda$ as expressed by the last Kronecker term.

The next step is to change the summation variables and rearrange the terms as suggested by Hulanicki in [15]. Let $\tilde{\rho}_{p}=\rho_{p}-\sigma_{p-1}=\left(\tilde{a}_{p}, \tilde{b}_{p}\right), 1 \leq p \leq L$, with convention $\sigma_{0}=(0,0)$. Also denote by $V_{p}$ the unitary

$$
\left(V_{p} s\right)_{\lambda=(t, \omega)}=e^{-i\left\langle\tilde{a}_{p}, \omega\right\rangle} s_{\lambda-\tilde{\rho}_{p}} .
$$

Then $c_{\lambda}$ turns into:

$$
\begin{aligned}
c_{\lambda}= & \sum_{\tilde{\rho}_{1}, \ldots, \tilde{\rho}_{L} \in \mathbf{R}^{2 d}} e^{i a_{1} b_{1}} r_{\tilde{\rho}_{1}}^{k_{1}} r_{\tilde{\rho}_{2}}^{k_{2}} \cdots r_{\tilde{\rho}_{L}}^{k_{L}} \sum_{\sigma_{1}, \ldots, \sigma_{L}}\left(V_{1} s^{j_{1}}\right)_{\sigma_{1}} \\
& \cdot e^{-i\left\langle c_{1}, d_{2}-d_{1}\right\rangle}\left(V_{2} s^{j_{2}}\right)_{\sigma_{2}-\sigma_{1}} e^{-i\left\langle c_{2}, d_{3}-d_{2}\right\rangle} \\
& \cdot \ldots \cdot e^{-i\left\langle c_{L-1}, d_{L}-d_{L-1}\right\rangle}\left(V_{L} s^{j_{L}}\right)_{\sigma_{L}-\sigma_{L-1}} \delta_{\sigma_{L}, \lambda} \\
= & \sum_{\tilde{\rho}_{1}, \ldots, \tilde{\rho}_{L} \in \mathbf{R}^{2 d}} e^{i a_{1} b_{1}} r_{\tilde{\rho}_{1}}^{k_{1}} r_{\tilde{\rho}_{2}}^{k_{2}} \cdots r_{\tilde{\rho}_{L}}^{k_{L}}\left(\left(V_{1} s^{j_{1}}\right) \sharp\left(V_{2} s^{j_{2}}\right) \sharp \cdots \sharp\left(V_{L} s^{j_{L}}\right)\right)_{\lambda}
\end{aligned}
$$

and thus

$$
\left|c_{\lambda}\right| \leq \sum_{\tilde{\rho}_{1}, \ldots, \tilde{\rho}_{L}}\left|r_{\tilde{\rho}_{1}}^{k_{1}}\right| \cdot \ldots \cdot\left|r_{\tilde{\rho}_{L}}^{k_{L}}\right| \cdot\left|\left(V_{1} s^{j_{1}} \sharp V_{2} s^{j_{2}} \sharp \cdots \sharp V_{L} s^{j_{L}}\right)_{\lambda}\right| .
$$

Since

$$
w(\lambda) \leq w\left(\tilde{\rho}_{1}\right) \cdot \ldots \cdot w\left(\tilde{\rho}_{L}\right) \cdot w\left(\lambda-\tilde{\rho}_{1}-\cdots-\tilde{\rho}_{L}\right)
$$

and

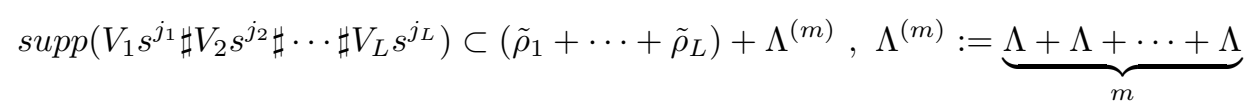

we obtain

$$
\left\|T^{n}\right\|_{\mathcal{A}_{v}} \leq \sum_{m=0}^{n}\left(\begin{array}{c}
n \\
m
\end{array}\right) \varepsilon^{n-m} \max _{\sigma \in \Lambda^{(m)}} v(\sigma) \sup _{\tilde{\rho}_{1}, \ldots, \tilde{\rho}_{L}} \sum_{\lambda}\left|\left(\left(V_{1} s^{j_{1}}\right) \sharp \cdots \sharp\left(V_{L} s^{j_{L}}\right)\right)_{\lambda}\right| .
$$

Now we will estimate the sum over $\lambda$ above similar to the estimation in (34). The cardinal of $\Lambda^{(m)}$ has been shown in Lemma 5.2 to be bounded by $(m+1)^{|\Lambda|}$ and hence by $(n+1)^{|\Lambda|}$. Note also that $\Lambda^{(m)} \subset E_{m R_{0}}(0)$ where $R_{0}$ is a radius so that $\Lambda \subset E_{R_{0}}(0)$. Thus we get:

$$
\begin{aligned}
\left\|T^{n}\right\|_{\mathcal{A}_{v} \leq} \leq(n+1)^{|\Lambda| / 2} w\left(n R_{0}\right) \sum_{m=0}^{n}\left(\begin{array}{c}
n \\
m
\end{array}\right) \varepsilon^{n-m} \\
\cdot \sup _{a_{1}, \ldots, a_{L}, b_{1}, \ldots, b_{L}}\left(\sum_{\lambda}\left|\left(\left(V_{1} s^{j_{1}}\right) \sharp \cdots \sharp\left(V_{L} s^{j_{L}}\right)\right)_{\lambda}\right|^{2}\right)^{1 / 2} .
\end{aligned}
$$

By Lemma 5.1 the $l^{2}$ norm of the sequence $s=\left(V_{1} s^{j_{1}} \sharp \cdots \sharp V_{L} s^{j_{L}}\right)$ is bounded by the operator norm obtained by linear combinations of time-frequency shifts with coefficients from $s$ :

$$
\|s\|_{2} \leq\left\|\sum_{\lambda} s_{\lambda} U_{\lambda}\right\|_{B\left(L^{2}\left(\mathbf{R}^{d}\right)\right)} .
$$

Note the operator associated to $s$ is (up to a constant phase factor):

$$
U_{\tilde{\rho}_{1}} T_{0}^{j_{1}} U_{\tilde{\rho}_{2}} T_{0}^{j_{2}} \cdot \ldots \cdot U_{\tilde{\rho}_{L}} T_{0}^{j_{L}} .
$$


Thus we get:

$$
\|s\|_{2} \leq\left\|T_{0}\right\|_{B\left(L^{2}\left(\mathbf{R}^{d}\right)\right)}^{j_{1}+\cdots+j_{L}}=\left\|T_{0}\right\|_{B\left(L^{2}\left(\mathbf{R}^{d}\right)\right)}^{m}
$$

which turns (47) into:

$$
\left\|T^{n}\right\|_{\mathcal{A}_{v}} \leq(n+1)^{|\Lambda|}\left(\varepsilon+\left\|T_{0}\right\|_{B\left(L^{2}\left(\mathbf{R}^{d}\right)\right)}\right)^{n} h .
$$

Now taking the $n^{\text {th }}$ root and passing to the limit $n \rightarrow \infty$ we obtain:

$$
r_{\mathcal{A}_{v}}(T) \leq \varepsilon+\left\|T_{0}\right\|_{B\left(L^{2}\left(\mathbf{R}^{d}\right)\right)} .
$$

Since $\varepsilon>0$ was arbitrary, and $\left\|T_{0}\right\| \leq\|T\|$ we obtain:

$$
r_{\mathcal{A}_{v}}(T) \leq\|T\|_{B\left(L^{2}\left(\mathbf{R}^{d}\right)\right)} .
$$

Since $r_{\mathcal{A}_{v}}\left(T^{n}\right)=\left(r_{\mathcal{A}_{v}}(T)\right)^{n}$ we obtain:

$$
r_{\mathcal{A}_{v}}(T) \leq\left\|T^{n}\right\|_{B\left(L^{2}\left(\mathbf{R}^{d}\right)\right)}^{1 / n}
$$

and passing to the limit $n \rightarrow \infty$ we obtain

$$
r_{\mathcal{A}_{v}}(T) \leq r_{B\left(L^{2}\left(\mathbf{R}^{d}\right)\right)}(T)
$$

The converse inequality is immediate from $\mathcal{A}_{v} \subset B\left(L^{2}\left(\mathbf{R}^{d}\right)\right)$. This ends the proof of (15) and hence of Theorem 3.1 .

Now Theorem 3.2 and subsequent corollaries are immediate. Theorem 3.2 and Corollary 3.3 are consequences of Hulanicki's Lemma (Proposition 2.5 in [17]; see also Proposition 9 in [12]).

During the proof of Theorem 3.1 we obtained the estimate (35). We will use this in proving Theorem 3.6.

Proof of Theorem 3.6. Assume $T$ is invertible in $B\left(L^{2}\left(\mathbf{R}^{d}\right)\right)$. Then for $A=\left\|T^{-1}\right\|^{-2}$ and $B=\|T\|^{2}$,

$$
0<A \leq T^{*} T \leq B<\infty
$$

Note $\left\|1-\frac{2}{A+B}\right\|=\frac{B-A}{B+A}<1$. Thus

$$
T^{-1}=\left(T^{*} T\right)^{-1} T^{*}=\frac{2}{A+B} \sum_{n \geq 0}\left(1-\frac{2}{A+B} T^{*} T\right)^{n} T^{*}
$$

which converges in operator norm in $B\left(L^{2}\left(\mathbf{R}^{d}\right)\right)$. The estimate (35) of Lemma 5.1] turns into:

$$
\left\|1-\frac{2}{A+B} T^{*} T\right\|_{\mathcal{A}_{v}} \leq(n+1)^{\left|\Lambda^{\prime}\right| / 2} w\left(n R_{0}^{\prime}\right)\left\|1-\frac{2}{A+B} T^{*} T\right\|^{n}
$$

where $\Lambda^{\prime}$ is the label set of $1-\frac{2}{A+B} T^{*} T$, and $R_{0}^{\prime}$ is so that $\Lambda^{\prime} \subset E_{R_{0}^{\prime}}(0)$. Since $\Lambda^{\prime} \subset \Lambda-\Lambda$ we have $\left|\Lambda^{\prime}\right| \leq 2|\Lambda|=2 N$ and $R_{0}^{\prime} \leq 2 R_{0}$, where $R_{0}=\max _{\lambda \in \Lambda}\|\lambda\|$ is so that $\Lambda \subset E_{R_{0}}(0)$. Thus we get

$$
\left\|T^{-1}\right\|_{\mathcal{A}_{v}} \leq \frac{2}{A+B} \sum_{n \geq 0}(n+1)^{N} w\left(2 n R_{0}\right)\left(\frac{B-A}{B+A}\right)^{n}\|T\|_{\mathcal{A}_{v}} .
$$

For $w(x)=C(1+x)^{m}$ and $\rho=\max \left(1,2 R_{0}\right), w\left(2 n R_{0}\right) \leq \rho^{m}(1+n)^{m}$ and for $\theta_{0}=(B-A) /(B+A)$ we obtain

$$
\left\|T^{-1}\right\|_{\mathcal{A}_{v}} \leq \frac{2 C \rho^{m}\|T\|_{\mathcal{A}_{v}}}{A+B} \sum_{n \geq 0}(1+n)^{m+N} \theta^{n} \leq\left.\frac{2 C \rho^{m}\|T\|_{\mathcal{A}_{v}}}{A+B}\left[\frac{d^{m+N}}{d \theta^{m+N}} \sum_{n \geq 0} \theta^{n}\right]\right|_{\theta=\theta_{0}} .
$$


Since $\theta_{0}<1$, by direct summation and then differentiation we obtain (17) which ends the proof of this theorem.

5.2. Proof of Theorems 3.7, 3.10 and Corollaries 3.8 and 3.9, For any bounded operator $T \in B\left(L^{2}\left(\mathbf{R}^{d}\right)\right)$ denote by $a_{M, N}(T)$ the following expression:

$$
a_{M, N}(T)=\frac{1}{(\alpha \beta)^{d}(2 M+1)^{d}(2 N+1)^{d}} \sum_{|m| \leq M} \sum_{|n| \leq N}\left\langle T g_{m, n ; \alpha, \beta}, \tilde{g}_{m, n ; \alpha, \beta}\right\rangle .
$$

Clearly $\left|a_{M, N}(T)\right| \leq C\|T\|_{B\left(L^{2}\left(\mathbf{R}^{d}\right)\right)}$, with $C$ independent of $T, M, N$.

Trace on $\mathcal{A}$

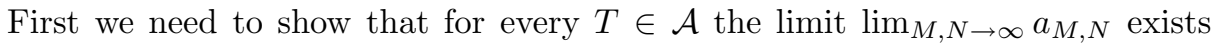
and equals $c_{0}=\gamma(T)$, the 0 -coefficient of $T$. We prove this statement in two steps. First we consider the unitary generators of $\mathcal{A}$, and then we extend by continuity to the entire $\mathcal{A}$.

Lemma 5.4. Let $U_{\lambda}$ denote the time-frequency shift with parameter $\lambda=(t, \omega)$. Then

$$
\lim _{M, N \rightarrow \infty} a_{M, N}\left(U_{\lambda}\right)=\left\{\begin{array}{lll}
1 & \text { if } \quad \lambda=0 \\
0 & \text { if } \quad \lambda \neq 0
\end{array}\right.
$$

Proof. We explicitly compute $a_{M, N}\left(U_{\lambda}\right)$

$$
\begin{aligned}
& a_{M, N}\left(U_{\lambda}\right)=\frac{1}{(\alpha \beta)^{2}(2 M+1)^{d}(2 N+1)^{d}} \\
& \cdot \sum_{|m| \leq M} \sum_{|n| \leq N} e^{2 \pi i m \alpha t} e^{i n \beta \omega} \int e^{i \omega x} g(x-t) \overline{\tilde{g}(x)} d x .^{\prime}
\end{aligned}
$$

There are now two cases:

Case 1. $(t, \omega)=\left(\frac{K}{\alpha}, \frac{2 \pi J}{\beta}\right)$ for some $K, J \in \mathbf{Z}^{d}$. Then $e^{2 \pi i m \alpha t}=e^{2 \pi i n \beta}=1$ and summations over $m$ and $n$ cancel the factor $(2 M+1)^{d}(2 N+1)^{d}$

$$
a_{M, N}\left(U_{\lambda}\right)=\int e^{\frac{2 \pi i}{\beta} J x} g(x-K \alpha) \overline{\tilde{g}(x)} d x .
$$

Recall $\mathcal{G}$ is a Gabor frame with dual frame $\tilde{\mathcal{G}}$. By duality principle $([\underline{6}$, [18], 27] $)$ $\mathcal{G}^{\prime}=\left\{g_{m, n ; \frac{1}{\beta}, \frac{1}{\alpha}} ; m, n \in \mathbf{Z}^{d}\right\}$ and $\tilde{\mathcal{G}}^{\prime}=\left\{\frac{1}{(\alpha \beta)^{d}} \tilde{g}_{m, n ; \frac{1}{\beta}, \frac{1}{\alpha}} ; m, n \in \mathbf{Z}^{d}\right\}$ are Riesz basic sequences biorthonormal to one another. Thus

$$
\left\langle g_{J, K ; \frac{1}{\beta}, \frac{1}{\alpha}}, \tilde{g}\right\rangle=(\alpha \beta)^{d} \delta_{J, 0} \delta_{K, 0}
$$

and combined with (54) proves (53) in this case.

Case 2. $\left(t \alpha, \frac{\omega \beta}{2 \pi}\right) \notin \mathbf{Z}^{2 d}$. Then for $t \alpha \notin \mathbf{Z}^{d}$ a direct computation shows

$$
\lim _{M \rightarrow \infty} \frac{1}{(2 M+1)^{d}} \sum_{|m| \leq M} e^{2 \pi i m \alpha t}=0
$$

whereas for $\frac{\omega \beta}{2 \pi} \notin \mathbf{Z}^{d}$

$$
\lim _{N \rightarrow \infty} \frac{1}{(2 N+1)^{d}} \sum_{|n| \leq N} e^{i n \beta \omega}=0 .
$$

This ends the proof of Lemma 5.4. 
By linearity we extend the result of this lemma to finite linear combinations of unitary operators $U_{\lambda}$ :

$$
\lim _{M, N \rightarrow \infty} a_{M, N}\left(\sum_{k=1}^{L} c_{\lambda_{k}} U_{\lambda_{k}}\right)=\left\{\begin{array}{rll}
c_{0} & \text { if } & 0 \in\left\{\lambda_{1}, \ldots, \lambda_{L}\right\} \\
0 & & \text { otherwise. }
\end{array}\right.
$$

Next the limit extends to the entire $\mathcal{A}$ by Lebesgue's dominated convergence theorem:

$$
\lim _{M, N \rightarrow \infty} a_{M, N}\left(\sum_{\lambda} c_{\lambda} U_{\lambda}\right)=\sum_{\lambda} c_{\lambda} \lim _{M, N \rightarrow \infty} a_{M, N}\left(U_{\lambda}\right)=c_{0}=\gamma\left(\sum_{\lambda} c_{\lambda} U_{\lambda}\right) .
$$

Consider $T=\sum_{\lambda} c_{\lambda} U_{\lambda} \in \mathcal{A}$ and $S=\sum_{\mu} d_{\mu} U_{\mu} \in \mathcal{A}$. Then $T S=\sum_{\rho}(c \sharp d)_{\rho} U_{\rho} \in \mathcal{A}$ and $S T=\sum_{\rho}(d \sharp c)_{\rho} U_{\rho} \in \mathcal{A}$. But now

$$
\gamma(T S)=(c \sharp d)_{0}=\sum_{\lambda} c_{\lambda} d_{-\lambda}=(d \sharp c)_{0}=\gamma(S T) .
$$

This shows $\gamma$ is a tracial state on $\mathcal{A}$. Finally, for $T=\sum_{\lambda} c_{\lambda} U_{\lambda} \in \mathcal{A}$. Then

$$
\gamma\left(T^{*} T\right)=\sum_{\lambda}\left|c_{\lambda}\right|^{2} \geq 0, \gamma\left(T^{*} T\right)=0 \text { iff } T=0
$$

Thus $\gamma$ is a faithful state.

Extension to A. When we set $g=\tilde{g}$ in (19), for any $T \in \mathcal{A}$ we obtain:

$$
|\gamma(T)| \leq\|T\|_{B\left(L^{2}\left(\mathbf{R}^{d}\right)\right)} .
$$

Then we can extend $\gamma$ to the completion of $\mathcal{A}$ with respect to the operator norm. The completion of $\mathcal{A}$ is denoted $\mathbf{A}$ and is a $C^{*}$ algebra. On this algebra, $\gamma$ remains a faithful tracial state. This ends the proof of Theorem 3.7

Corollaries of Theorem 3.7. The proof of Corollary 3.8 is immediate. In particular equation (20) follows as in (57), whereas (21) is a consequence of Lemma 5.4 .

For proving Corollary 3.9 note first that if $T$ is a finite rank operator, then $\operatorname{trace}\left(T^{*} T\right)<\infty$. But then

$$
\operatorname{trace}\left(T^{*} T\right)=\sum_{m, n \in \mathbf{Z}^{d}}\left\langle T^{*} T g_{m, n ; \alpha, \beta}, \tilde{g}_{m, n ; \alpha, \beta}\right\rangle<\infty
$$

and thus $\lim _{M, N \rightarrow \infty} a_{M, N}\left(T^{*} T\right)=0$. By Corollary 3.8 this implies $T=0$. Now consider $T$ a compact operator in $\mathbf{A}$. Then $T^{*} T \in \mathbf{A}$ is a nonnegative compact operator. Let $s>0$ be an eigenvalue of $T^{*} T$ and let $P_{s}$ denote the projection onto its eigenspace. On the one hand $P_{s}$ is finite rank, since $T^{*} T$ is compact; on the other hand, by holomorphic functional calculus (see [25]), $P_{s}$ belongs to the $C^{*}$ algebra A. Then, as shown before, $P_{s}$ has to vanish, which proves $T=0$.

Proof of Theorem 3.10 . Theorem 3.10 is a consequence of the holomorphic functional calculus. Assume $T=\sum_{\lambda} c_{\lambda} U_{\lambda}$ has a finite isolated eigenvalue, say $\mu_{0}$. Since it is isolated, by holomorphic functional calculus (see [25]) the orthogonal projection onto the eigenspace is given by

$$
P_{\mu_{0}}=\frac{1}{2 \pi i} \int_{\Gamma}(z I-T)^{-1} d z
$$

where $\Gamma$ is a circle in the complex plan centered at $\mu_{0}$ so that it separates $\mu_{0}$ from the rest of the spectrum of $T$. Thus $P_{\mu_{0}} \in \mathbf{A}$. Since $\mu_{0}$ has finite multiplicity it 
follows that $P_{\mu_{0}}$ has finite rank, but then by Corollary 3.9, $P_{\mu_{0}}=0$, which ends the proof of Theorem 3.10 .

5.3. Proof of Theorems 3.12 and 3.14. Theorems 3.12 and 3.14 characterize finite and half-compactly supported operators in $\mathcal{A}$. The proof of Theorem 3.12 is based on a spectral radius computation done in Lemma 5.1. In turn, Theorem 3.12 allows the operator extension to the Banach space $L^{2, \infty}$ introduced in Section 3. Once this extension is established, Theorem 3.14 follows easily.

For a $\rho>0$ we set $f(\lambda)=e^{\rho\|\lambda\|}, \lambda \in \mathbf{R}^{d}$. For convenience we denote $\mathcal{B}_{\rho}=\mathcal{A}_{f}$, the Banach algebra of bounded operators in $\mathcal{A}$ whose coefficients decay exponentially fast with rate $\rho$. Note the spectral radius of an operator $T \in \mathcal{B}_{\rho}$ is not the same as the spectral radius in $B\left(L^{2}\left(\mathbf{R}^{d}\right)\right)$, since $f$ does not satisfy the GRS condition.

Proof of Theorem 3.12, Assume $T=\sum_{\lambda \in \Lambda} c_{\lambda} U_{\lambda}$ is an invertible operator on $L^{2}\left(\mathbf{R}^{d}\right)$ with finite support. Let $A, B>0$ be the bounds in

$$
A\|f\|^{2} \leq\|T f\|^{2} \leq B\|f\|^{2}, \forall f \in L^{2}\left(\mathbf{R}^{d}\right) .
$$

Then

$$
T^{-1}=\frac{2}{A+B} \sum_{n \geq 0} R^{n} T^{*}
$$

where $R=1-\frac{2}{A+B} T^{*} T$. Since $R$ has finite support let $N:=|\operatorname{supp}(R)|<\infty$ and $R_{0}>0$ so that $\operatorname{supp}(R) \subset E_{R_{0}}(0)$.

The goal is to show there exists a $\rho>0$ so that $T^{-1} \in \mathcal{B}_{\rho}$. Clearly each term in (59) belongs to $\mathcal{B}_{\rho}$. The only problem is to check that the series converges in $\mathcal{B}_{\rho}$. To do so it is sufficient to show that

$$
\limsup _{n \rightarrow \infty}\left(\left\|R^{n}\right\|_{\mathcal{B}_{\rho}}\right)^{1 / n}<1
$$

Let us choose $\rho=\frac{1}{R_{0}} \ln \frac{B+A}{2(B-A)}$. Then $e^{\rho R_{0}}\|R\| \leq \frac{1}{2}$. Now we apply Lemma 5.1 to the algebra $\mathcal{B}_{\rho}$, since $f(x)=e^{\rho\|x\|}$ is a submultiplicative weight. We obtain:

$$
\left(\left\|R^{n}\right\|_{\mathcal{B}_{\rho}}\right)^{1 / n} \leq(n+1)^{N /(2 n)} e^{\rho R_{0}}\|R\| .
$$

By choice of $\rho$ we obtain (60) which ends the proof of Theorem 3.12

Let us now turn to Theorem 3.14. First we recall the mixed norm Banach space introduced in Section. 3, For a compact neighborhood of the origin $I$ we denote by $L^{2, \infty}\left(\mathbf{R}^{d} \times I\right)$ the mixed norm Banach space defined in (25). An operator $T=\sum_{\lambda \in \Lambda} c_{\lambda} U_{\lambda} \in \mathcal{A}$ extends from $L^{2}\left(\mathbf{R}^{d}\right)$ to $L^{2, \infty}\left(\mathbf{R}^{d} \times I\right)$ by

$$
f \in L^{2, \infty}\left(\mathbf{R}^{d} \times I\right) \mapsto \mathbf{T} f(x, y)=\sum_{\lambda=(t, \omega) \in \Lambda} c_{\lambda} e^{i\langle\omega, x+i y\rangle} f(x-t, y), \forall(x, y) \in \mathbf{R}^{d} \times I .
$$

Assume that for all $\lambda=(t, \omega) \in \Lambda,\|\omega\| \leq \Omega$; in other words $\Lambda \subset \mathbf{R}^{d} \times E_{\Omega}(0)$. Assume also $I \subset E_{\rho}(0)$, for some $\rho>0$. Then

$$
\|\mathbf{T} f\|_{2, \infty} \leq \sum_{\lambda \in \Lambda} \sup _{y \in I} e^{-\langle\omega, y\rangle}\left(\int_{\mathbf{R}^{d}}|f(x, y)|^{2} d x\right)^{1 / 2} \leq e^{\rho \Omega} \sum_{\lambda}\left|c_{\lambda}\right|\|f\|_{2, \infty} .
$$

Thus

$$
\|\mathbf{T}\|_{B\left(L^{2, \infty}\left(\mathbf{R}^{d} \times I\right)\right)} \leq e^{\rho \Omega}\|T\|_{\mathcal{A}}
$$


This proves $T$ extends to a bounded operator $\mathbf{T}$ on $L^{2, \infty}\left(\mathbf{R}^{d} \times I\right)$. Let $T^{(y)}$ denote the restriction of $\mathbf{T}$ to the "slice" indexed by $y$ of $L^{2, \infty}$ :

$$
T^{(y)}: L^{2}\left(\mathbf{R}^{d}\right) \rightarrow L^{2}\left(\mathbf{R}^{d}\right), T^{(y)} f(x)=\sum_{\lambda=(t, \omega)} c_{\lambda} e^{-\langle\omega, y\rangle} U_{\lambda} f(x) .
$$

Note for $\Lambda \in \mathbf{R}^{d} \times E_{\Omega}(0)$ each $T^{(y)} \in \mathcal{A}$ and

$$
\|\mathbf{T}\|_{L^{2, \infty}\left(\mathbf{R}^{d} \times I\right)}=\sup _{y \in I}\left\|T^{(y)}\right\|_{B\left(L^{2}\left(\mathbf{R}^{d}\right)\right)} .
$$

Now we are ready to prove Theorem 3.14 .

Proof of Theorem 3.14. (1) The estimate (28) follows from (62).

(2) From (63), (64) and Corollary 3.8 we get:

$$
e^{-\langle\omega, y\rangle}\left|c_{\lambda}\right| \leq\left\|T^{(y)}\right\|_{B\left(L^{2}\left(\mathbf{R}^{d}\right)\right)} \leq\|T\|_{B\left(X_{\rho}\right)} .
$$

Combine now with (28) to obtain:

$$
\left|c_{\lambda}\right| \leq C e^{\rho \Omega+\langle\omega, y\rangle} .
$$

Assume there is a $\lambda=(t, \omega) \in \Lambda$ so that $\|\omega\|>\Omega$. Choose $y \in E_{\rho}(0)$ so that $\langle\omega, y\rangle=-\rho\|\omega\|$. Then

$$
\left|c_{\lambda}\right| \leq C e^{-\rho(\|\omega\|-\Omega)}
$$

should hold valid for all $\rho>0$. At limit $\rho \rightarrow \infty$ we get $\left|c_{\lambda}\right| \leq 0$ which proves that $\operatorname{supp}(T) \subset \mathbf{R}^{d} \times E_{\Omega}(0)$.

(3) and (4) follows from (1) and (2) by noticing that the Fourier transform is an intertwining operator between the translation shift and the modulation shift that switches the modulation and translation parameters. Thus (3) and (4) reduce to (1) and (2).

\section{ACKNOWLEDGMENTS}

The author wishes to thank Thomas Strohmer for pointing out the papers by Sjöstrand [28], Gohberg [8], and then for comments on using this approach to a class of pseudodifferential operators. The trace results would not have been possible without the stimulating discussions the author had with Zeph Landau. The author is very happy to acknowledge and thank him for all the comments and suggestions received during the past seven years. Many thanks go to Chris Heil for discussions and ideas exchanged during all these years. Special thanks go to Charly Gröchenig for discussions on the approach used in [12, and to Hans Feichtinger for comments on the draft. The author also thanks Hans Feichtinger, Charly Gröchenig, John Benedetto, and the Erwin Schrödinger Institute for the invitation and hospitality shown to him during his stay in Vienna at ESI in May 2005, when most of the proofs of this paper were obtained.

\section{REFERENCES}

1. R. Balan, P. Casazza, C. Heil, and Z. Landau, Deficits and Excesses of Frames, Advances in Computational Mathematics 18 (2003), 93-116. MR.1968114(2004a:42040)

2. _ Excesses of Gabor Frames, Appl. Comput. Harmon. Anal. 14 (2003), 87-106. MR 1981203 (2004c:42058)

3. , Density, Overcompleteness, and Localization of Frames. I. Theory, J. Fourier Anal. Applic. 12 (2006), no. 2, 105-143. MR2224392 (2007b:42041) 
4. _ Density, Overcompleteness, and Localization of Frames. II. Gabor Frames, J. Fourier Anal. Applic. 12 (2006), no. 3, 309-344. MR2235170 (2007b:42042)

5. A.G. Baskakov, Estimates for the entries of inverse matrices and the spectral analysis of linear operators, Izvestiya: Mathematics 61 (1997), no. 6, 1113-1135. MR.1609144 (98m:47007)

6. I. Daubechies, H. Landau, and Z. Landau, Gabor Time-Frequency Lattices and the WexlerRaz Identity, J. Fourier Analys. Applic. 1 (1995), no. 4, 437-478. MR1350701 (96i:42021)

7. M. Fornasier and K. Gröchenig, Intrinsic Localization of Frames, Constructive Approximation 22 (2005), no. 3, 395-415. MR2164142 (2006f:42030)

8. I. Gohberg, M.A. Kaashoek, and H.J. Woerdeman, The band method for positive and strictly contractive extension problems: an alternative version and new applications, Integral Equations and Operator Theory 12 (1989), 343-382. MR998278 (90c:47022)

9. K. Gröchenig, Foundations of Time-Frequency Analysis, Birkhäuser Boston, Inc., 2001.

10. - Localization of Frames, Banach Frames, and the Invertibility of Frame Operator, J. Fourier Anal. Applic. 10 (2004), 105-132. MR2054304 (2005f:42086)

11. 703-724. MR2294795

12. K. Gröchenig and M. Leinert, Wiener's Lemma for Twisted Convolution and Gabor Frames, Journal of AMS 17 (2003), no. 1, 1-18.

13. Symmetry of Matrix Algebras and Symbolic Calculus for Infinite Matrices, Trans. of AMS 358 (2006), no. 6, 2695-2711. MR2204052(2006k:47065)

14. C. Heil, Linear Independence of Finite Gabor Systems, "Harmonic Analysis and Applications", a volume in honor of John J. Benedetto's 65th birthday (C. Heil, ed.), Birkhäuser, 2005. MR2249310 (2007d:42057)

15. A. Hulanicki, On the spectral radius of hermitian elements in group algebras, Pacific J. Math. 18 (1966), 277-287. MR0198267 (33:6426)

16. On symmetry of group algebras of discrete nilpotent groups, Studia Math. 35 (1970), 207-219. MR0278082 (43:3814)

17. - On the spectrum of convolution operators on groups with polynomial growth, Invent. Math. 17 (1972), 135-142. MR0323951 (48:2304)

18. A.J.E.M. Janssen, Duality and Biorthogonality for Weyl-Heisenberg Frames, J. Fourier Anal. Applic. 1 (1995), no. 4, 403-436. MR1350700 (97e:42007)

19. P.A. Linnell, Von Neumann algebras and linear independence of translates, Proc. AMS $\mathbf{1 2 7}$ (1999), 3269-3277. MR1637388 (2000b:46106)

20. L.H. Loomis, An Introduction to Abstract Harmonic Analysis, D. van Nostrand Company, Inc., 1953. MR0054173(14:883c)

21. J. Ludwig, A class of symmetric and a class of Wiener group algebras, J. Funct. Anal. 31 (1979), no. 2, 187-194. MR.525950 (81a:43007)

22. M.A. Neimark, Normed algebras, Wolters-Noordhoff Publishing, Groningen, third edition, 1972. MR 0438123 (55:11042)

23. C.E. Rickart, General theory of Banach algebras, D. van Nostrand Co., Inc., 1960. MR0115101 $(22: 5903)$

24. M.A. Rieffel, Von Neumann Algebras Associated with Pairs of Lattices in Lie Groups, Math. Anal. 257 (1981), 403-418. MR639575 (84f:22010)

25. F. Riesz and B.Sz. Nagy, Functional Analysis, Dover Publications, New York, 1990. MR.1068530 (91g:00002)

26. L. Rodman, I.M. Spitkovsky, and H.J. Woerdeman, Carathéodory-Toeplitz and Nehari Problems for Matrix Valued Almost Periodic Functions, Trans. AMS 350 (1998), no. 6, 2185-2227. MR 1422908 (98h:47023)

27. A. Ron and Z. Shen, Weyl-Heisenberg frames and Riesz bases in $L_{2}\left(\mathbf{R}^{\mathbf{d}}\right)$, Duke Math. J. 89 (1997), no. 2, 237-282. MR1460623 (98i:42013)

28. J. Sjöstrand, Wiener type algebras of pseudodifferential operators, Séminaire sur les Equations aux Dérivées Partielles, 1994-1995, École Polytech., Palaiseau 21 (1995), Exp. No. IV. MR $1362552(96 \mathrm{j}: 47049)$

29. T. Strohmer, Pseudodifferential operators and Banach algebras for mobile communications, Appl. Comp. Harmon. Anal. 20 (2006), no. 2, 237-249. MR2207837 
30. - personal communication (September 2005).

31. A. Zygmund, Trigonometric Series, University Press, Cambridge, 3rd Edition, 2003.

Siemens Corporate Research, 755 College Road East, Princeton, New Jersey 08540 E-mail address: radu.balan@siemens.com

Current address: Department of Mathematics, University of Maryland, College Park, Maryland 20742

E-mail address: rvbalan@math.umd.edu 